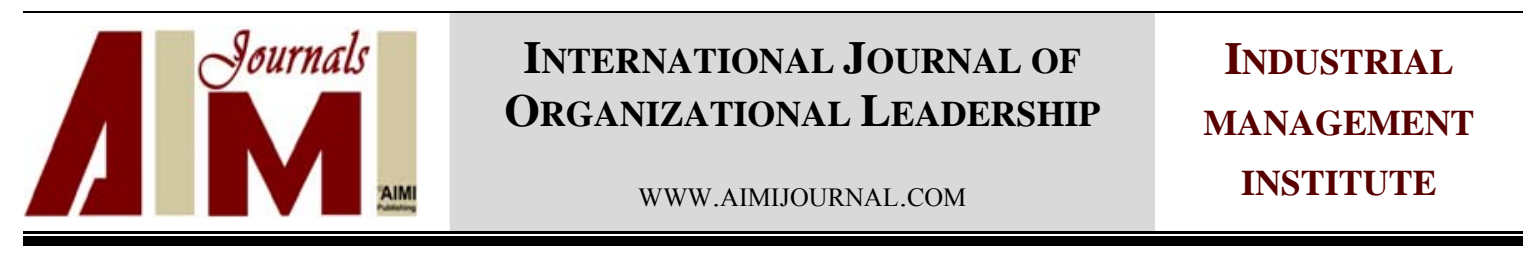

\title{
Identification and comparison of structural factors of innovation capability in ESCO with desirable status
}

\author{
Fatemeh Jalali*, Abbas Khamseh \\ EMBA student, Science and Research University, Karaj, Iran \\ Department of Industrial Management, Islamic Azad University, Karaj, Iran
}

\begin{abstract}
Keywords:

The present study describes the identification and comparison of structural factors of innovation capability in Esfahan Steel Company (ESCO). Innovation is a crucial factor in growth, success, and survival of organizations. Since the innovation for organizations is not possible without the level of innovation capabilities and the need for steel products and

innovation, innovation capability, the existing situation, the desired situation imports of goods from developed countries has greatly increased, this study intends to investigate the factors affecting the subject that may be able to increase the production and reduce the need to import it. Evaluation of the innovation capability factors of ESCO compared with its desired status in industry can help companies develop innovative strategies

Correspondence: and also achieve organizational goals. Statistical analysis methods and mean comparison test by examining the structure of the innovation capability in the form of a standard questionnaire

Jalali.mahour@gmail.com was employed. The findings suggest that the innovation capability in the existing situation of ESCO in comparison with the desired situation is significantly different.
\end{abstract}

CAIMI Journals

\section{Introduction}

Khalil (2013) believes that innovation involves various information, imagination, and initiative to provide a new product, service, or process to the market through the creation of new applications of existing technologies or the creation and commercialization of new technologies. In a similar vein, he argues that innovation is a kind of competitive weapon. 
Innovation capability is the ability to use the technology and scale of innovation in organizations, companies, and various agencies. Since innovation can be very large and complex, reviewing it in separate parts can result in better outcome. In this study, we investigated one of the innovation infrastructures including innovation capability and its indexes in the steel industry and specifically in ESCO.

\section{Assessing Innovation Capability}

The process of globalization has influenced every aspect of competing, resulting in more competitions over resources and markets. Organizations compete for intangible assets such as human resources. It is evident that competition for the market by using supply of high quality products has increased. Simply put, innovation is the process of taking what is outdated, reviving it as a form of improvement, and renewing the organization's products or services in external environment. In fact, the innovation capability is a prerequisite for realization of innovation in enterprises. Assessing the innovation capability provides unique opportunity to analyze the situation, the potential of each firm, and comparison with the competitors for making strategic decisions.

In assessment, as a literal means to determine the value of something, there is an agreement that assessment does analyze the efficiency and cost of the project after its implementation. If assessment is performed within the scope of the implementation plan, its aims will be improvement and management of the programs or decision making to continue, extend, or revise the plan. Assessment also means the realization of the value and cost of things. Innovation capability and its assessment can help us find the place of our organization in different environmental conditions and today's complex market.

Since the innovation capability refers to the ability of domestic firms for industrial innovation systematically and follows it to achieve or increase a competitive advantage, innovation capability assessment might play a key role in the growth and survival of organizations. Accordingly, many of the rapid changes occurring in the economic systems are now understood by assessing new technologies and innovations (Kroll \& Schiller, 2010).

Nowadays, scientific and industrial communities have come to the conclusion that organizations with a focus on innovation, fostering, promoting innovation and innovative activities within their own, can maintain their long-term honors in competitive field. Also, they can be known as the main aspects and means of transforming an idea into an opportunity to solve the problems ahead (Saunila, Ukko, \& Rantanen, 2014). 
In the past centuries, Iranians discovered how to obtain iron and steel. Also, in the last three centuries, the speed of the development in technology and in steel production in industrialized countries has increased, so we have more demand for steel products.

As shown by the vision and plans of the industry, market, and companies, it seems that we need more and more company products and also we are in need of upgrading the technology used in industry. So, innovation, as an essential factor, can be taken into account in this sector. Besides, the innovation capability refers to ability of domestic firms for industrial innovation systematically and follows it to achieve or increase a competitive advantage, and according to the explanation given and evaluation of innovation capability in organization, the ability is considered as a core of strategy of innovation and technology. Therefore, investigating this topic and discovering the strengths and weaknesses of the organization in this case can be very helpful in management decisions related to the topic.

\section{Literature Review}

Tidd and Bessant (2009) argues that innovation is more than just good ideas; it is the process of nurturing ideas and changing them to practical forms which could be used to take advantage of them. He believes that although different words are used in the definitions of innovation, but they all emphasized the need for completing the development and exploitation of new knowledge. Potential of a firm's innovative activities have called "innovation capability", including new products and services, processes or procedures, and new ideas about the organization (Arasti, Karamipour, \& Quraishi, 2009). Understanding the innovation capability and assessing its productive and affecting factors in organization can be used in the development and exploitation of existing and available knowledge and technology and then develop the appropriate strategy. Based on the studies conducted to date, the following table is extracted which can be used for recognizing the productive factors of innovation capability.

Table 1

Literature Review

\begin{tabular}{clc}
\hline \multicolumn{1}{c}{ Studies } & \multicolumn{1}{c}{ Indexes } \\
\hline Dimensions & \multicolumn{1}{c}{ Khalil (2000) } & Personal knowledge \\
& Roman, Gamero, \& Tamayo (2011), Forsman (2011) & \\
& Kroll and Schiller (2010), Akman and Yilmaz (2008) & Personal talent \\
Individual & Yang (2012), Roman, Gamero, \& Tamayo (2011) & \\
& Hull and Covin (2010), Kroll and Schiller (2010) & \\
& Nematolahi (2011), Roman, Gamero, \& Tamayo (2011) & Ability and effort for innovative ideas \\
& Hull \& Covin (2010), Akman \& Yilmaz (2008), & $\begin{array}{c}\text { Staff Information of the status of innovation in } \\
\text { organizations } \\
\text { Kroll \& Schiller (2010) }\end{array}$ \\
& Roman, Gamero, \& Tamayo (2011), Forsman (2011), Akman \& & Yilmaz (2008) \\
\hline
\end{tabular}




\begin{tabular}{|c|c|c|}
\hline Team & Bertland (2009) & $\begin{array}{c}\text { Communication between colleagues in team } \\
\text { Communication between the teams } \\
\text { Conformity of people in a team }\end{array}$ \\
\hline \multirow{28}{*}{ Organizational } & Yeşil (2013), Roman, Gamero, \& Tamayo (2011) & The orientation towards innovation strategies \\
\hline & Yang (2012) & Promotion and reward systems \\
\hline & Yang (2012) & Punishment systems \\
\hline & Bertland (2009), Akman \& Yilmaz (2008), & Leadership and performance of managers \\
\hline & Nematolahi (2011), Bertland (2009) & The support of innovators \\
\hline & Akman \& Yilmaz (2008) & Speed of managers in the process of innovation \\
\hline & Yeșil (2013), Forsman (2011) & Dependence on other sources \\
\hline & Nematolahi (2011) & \\
\hline & Roman, Gamero, \& Tamayo (2011) & Organizational structure \\
\hline & Roman, Gamero, \& Tamayo (2011) & Organizational goals \\
\hline & Yeşil (2013), Yang (2012) & Learning \\
\hline & Roman, Gamero, \& Tamayo (2011), Hull \& Covin (2010) & \\
\hline & Yeşil (2013), Pakzad and Tabatabaeian (2005) & Internal R\&D spending and their nature \\
\hline & Yeşil (2013), Nematolahi (2011) & R\&D budget \\
\hline & Pakzad and Tabatabaeian (2005), Kroll \& Schiller (2010), & \\
\hline & Bertland (2009) & \\
\hline & Nematolahi (2011) & Rules and regulations \\
\hline & Roman, Gamero, \& Tamayo (2011) & Organizational culture \\
\hline & Roman, Gamero, \& Tamayo (2011) & Growth and maturity of organization \\
\hline & Nematolahi (2011), Roman, Gamero, \& Tamayo (2011) & Resource \\
\hline & Forsman (2011), Bertland (2009) & \\
\hline & Roman, Gamero, \& Tamayo (2011) & Knowledge management \\
\hline & Arasti, Karamipour, \& Quraishi (2009) & $\begin{array}{c}\text { Individual and team performance evaluation } \\
\text { systems }\end{array}$ \\
\hline & Arasti, Karamipour, \& Quraishi (2009) & System performance evaluation based on goals \\
\hline & Yeşil (2013) & Creativity in methods of operation \\
\hline & Shekarchizadeh (2011) & \\
\hline & Yeşil (2013), Forsman (2011) & Number of patents \\
\hline & Nematolahi (2011), Bertland (2009) & $\begin{array}{l}\text { Time allocated for innovative ideas } \\
\text { Resources allocated to the process of innovation }\end{array}$ \\
\hline \multirow{12}{*}{ National } & Shekarchizadeh (2011) & National standards \\
\hline & Nematolahi (2011) & \\
\hline & Shekarchizadeh (2011) & Public laws and regulations protecting \\
\hline & $\begin{array}{l}\text { Shekarchizadeh (2011), Bertland (2009), Akman and Yilmaz } \\
\text { (2008) }\end{array}$ & Customer affect \\
\hline & Shekarchizadeh (2011) & Supplier affect \\
\hline & Bertland (2009), Akman and Yilmaz (2008), & \\
\hline & Shekarchizadeh (2011) & Cooperation with other companies \\
\hline & Bertland (2009), Akaman and Yilmaz (2008) & \\
\hline & Shekarchizadeh (2011), Bertland (2009) & Economic conditions in the community \\
\hline & Shekarchizadeh (2011), Bertland (2009) & Political conditions in the community \\
\hline & Forsman (2011), Bertland (2009) & Using the previous knowledge and experience \\
\hline & Pakzad and Tabatabaeian (2005) & \\
\hline
\end{tabular}

According to the studies to date, structural determinants of innovation capability and index for each of them were obtained. The validity of the obtained structure through the use of exploratory factor analysis was determined. Then, the associated conceptual model was approved. In sum, this study is an attempt to evaluate the factors and criteria in the relevant company.

\section{Research Question and Hypotheses}

The following research question guided this study:

At which level is each of innovation capability factors located? 
Also, the following research hypotheses were postulated:

$\mathrm{H}_{0}$ : Each factor of innovation capabilities in ESCO is in desirable level.

$\mathrm{H}_{1}$ : Each factor of innovation capabilities in ESCO is not in desirable level.

However, the gap between existing and desired status was investigated by the following hypothesis.

$$
\left\{\begin{array}{l}
H_{0}: \mu_{\text {existing }}=\mu_{\text {desired }} \\
H_{1}: \mu_{\text {existing }} \neq \mu_{\text {desired }}
\end{array}\right.
$$

Table 2 contains the results of the comparison test between the two statuses. (t-test)

Table 2

Paired t-test Analysis of Innovation Capability

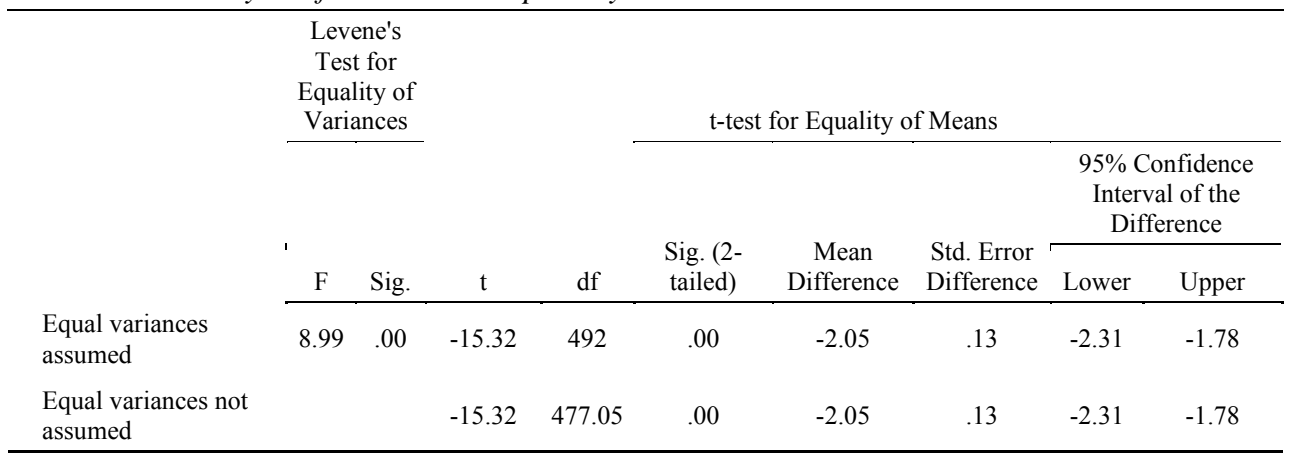

Therefore, it can be argued that the innovation capability has a significant difference in the existing situation to desired situation. Due to the lower limit $(-2.31)$ and upper limit $(-1.78)$ it can be argued that the existing situation is unfavorable.

To this end, this study evaluates the existing status of innovation capability's factor with desired to find the gap between them. The results of the comparison test between two statuses are shown in Table 3. At first, we examined the equality of two population variances.

Table 3

Comparative Analysis of Innovation Capability Indexes

\begin{tabular}{|c|c|c|c|c|c|c|c|c|c|c|}
\hline \multirow{4}{*}{$x 1$} & & \multicolumn{2}{|c|}{$\begin{array}{c}\text { Levene's Test for } \\
\text { Equality of } \\
\text { Variances }\end{array}$} & \multicolumn{7}{|c|}{ t-test for Equality of Means } \\
\hline & & \multirow[t]{2}{*}{$\mathrm{F}$} & \multirow[t]{2}{*}{ Sig. } & \multirow[t]{2}{*}{$\mathrm{t}$} & \multirow[t]{2}{*}{ df } & \multirow[t]{2}{*}{$\begin{array}{c}\text { Sig. } \\
\text { (2-tailed) }\end{array}$} & \multirow[t]{2}{*}{$\begin{array}{c}\text { Mean } \\
\text { Difference }\end{array}$} & \multirow[t]{2}{*}{$\begin{array}{l}\text { Std. Error } \\
\text { Difference }\end{array}$} & \multicolumn{2}{|c|}{$\begin{array}{l}95 \% \text { Confidence } \\
\text { Interval of the } \\
\text { Difference }\end{array}$} \\
\hline & & & & & & & & & Lower & Upper \\
\hline & $\begin{array}{l}\text { Equal variances } \\
\text { not assumed }\end{array}$ & & & -8.06 & 460.90 & .000 & -1.38 & .17 & -1.72 & -1.04 \\
\hline$x 2$ & $\begin{array}{c}\text { Equal variances } \\
\text { assumed }\end{array}$ & 27.59 & .000 & -8.16 & 491 & .000 & -1.50 & .18 & -1.86 & -1.14 \\
\hline
\end{tabular}




\begin{tabular}{|c|c|c|c|c|c|c|c|c|c|c|}
\hline & & \multicolumn{2}{|c|}{$\begin{array}{c}\text { Levene's Test for } \\
\text { Equality of } \\
\text { Variances }\end{array}$} & \multicolumn{7}{|c|}{ t-test for Equality of Means } \\
\hline & & \multirow[t]{2}{*}{$\mathrm{F}$} & \multirow[t]{2}{*}{ Sig. } & \multirow[t]{2}{*}{$\mathrm{t}$} & \multirow[t]{2}{*}{$\mathrm{df}$} & \multirow[t]{2}{*}{$\begin{array}{c}\text { Sig. } \\
\text { (2-tailed) }\end{array}$} & \multirow[t]{2}{*}{$\begin{array}{c}\text { Mean } \\
\text { Difference }\end{array}$} & \multirow[t]{2}{*}{$\begin{array}{l}\text { Std. Error } \\
\text { Difference }\end{array}$} & \multicolumn{2}{|c|}{$\begin{array}{c}95 \% \text { Confidence } \\
\text { Interval of the } \\
\text { Difference }\end{array}$} \\
\hline & & & & & & & & & Lower & Upper \\
\hline & $\begin{array}{c}\text { Equal variances } \\
\text { not assumed }\end{array}$ & & & -8.17 & 452.81 & .000 & -1.50 & .18 & -1.86 & -1.14 \\
\hline \multirow[t]{2}{*}{$x 3$} & $\begin{array}{c}\text { Equal variances } \\
\text { assumed }\end{array}$ & 17.00 & .000 & -9.27 & 490 & .000 & -1.75 & .18 & -2.12 & -1.38 \\
\hline & $\begin{array}{c}\text { Equal variances } \\
\text { not assumed }\end{array}$ & & & -9.28 & 465 & .000 & -1.75 & .18 & -2.12 & -1.38 \\
\hline \multirow[t]{2}{*}{$x 4$} & $\begin{array}{c}\text { Equal variances } \\
\text { assumed }\end{array}$ & 19.66 & .000 & -4.35 & 491 & .000 & -.77 & .17 & -1.13 & -.42 \\
\hline & $\begin{array}{c}\text { Equal variances } \\
\text { not assumed }\end{array}$ & & & -4.36 & 4.38 & .000 & -.77 & .17 & -1.13 & -.42 \\
\hline \multirow[t]{2}{*}{$x 5$} & $\begin{array}{c}\text { Equal variances } \\
\text { assumed }\end{array}$ & 8.10 & .005 & -5.74 & 485 & .000 & -1.09 & .19 & -1.47 & -.72 \\
\hline & $\begin{array}{c}\text { Equal variances } \\
\text { not assumed }\end{array}$ & & & -5.76 & 473.79 & .000 & -1.09 & .19 & -1.47 & -.72 \\
\hline \multirow[t]{2}{*}{$x 6$} & $\begin{array}{c}\text { Equal variances } \\
\text { assumed }\end{array}$ & 14.23 & .000 & -10.66 & 488 & .000 & -2.16 & .20 & -2.56 & -1.76 \\
\hline & $\begin{array}{c}\text { Equal variances } \\
\text { not assumed }\end{array}$ & & & -10.67 & 469.14 & .000 & -2.16 & .20 & -2.56 & -1.76 \\
\hline \multirow[t]{2}{*}{$x 7$} & $\begin{array}{c}\text { Equal variances } \\
\text { assumed }\end{array}$ & 4.70 & .031 & 3.30 & 488 & .001 & .82 & .24 & .33 & 1.31 \\
\hline & $\begin{array}{c}\text { Equal variances } \\
\text { not assumed }\end{array}$ & & & 3.30 & 478.03 & .001 & .82 & .24 & .33 & 1.31 \\
\hline \multirow[t]{2}{*}{$x 8$} & $\begin{array}{c}\text { Equal variances } \\
\text { assumed }\end{array}$ & .35 & .551 & -8.83 & 489 & .000 & -1.77 & .20 & -2.17 & -1.38 \\
\hline & $\begin{array}{c}\text { Equal variances } \\
\text { not assumed }\end{array}$ & & & -8.83 & 487.98 & .000 & -1.77 & .20 & -2.16 & -1.38 \\
\hline \multirow[t]{2}{*}{$x 9$} & $\begin{array}{c}\text { Equal variances } \\
\text { assumed }\end{array}$ & 3.04 & .081 & -8.56 & 489 & .000 & -1.59 & .18 & -1.95 & -1.22 \\
\hline & $\begin{array}{c}\text { Equal variances } \\
\text { not assumed }\end{array}$ & & & -8.56 & 483.55 & .000 & -1.59 & .18 & -1.95 & -1.22 \\
\hline \multirow[t]{2}{*}{$x 10$} & $\begin{array}{c}\text { Equal variances } \\
\text { assumed }\end{array}$ & 15.72 & .000 & -7.51 & 491 & .000 & -1.29 & .17 & -1.62 & -.95 \\
\hline & $\begin{array}{c}\text { Equal variances } \\
\text { not assumed }\end{array}$ & & & -7.51 & 459.901 & .000 & -1.29 & .17 & -1.62 & -.95 \\
\hline \multirow[t]{2}{*}{$x 11$} & $\begin{array}{l}\text { Equal variances } \\
\text { assumed }\end{array}$ & 2.71 & .100 & -7.02 & 492 & .000 & -1.28 & .18 & -1.64 & -.92 \\
\hline & $\begin{array}{l}\text { Equal variances } \\
\text { not assumed }\end{array}$ & & & -7.02 & 486.30 & .000 & -1.28 & .18 & -1.64 & -.92 \\
\hline \multirow[t]{2}{*}{$x 12$} & $\begin{array}{c}\text { Equal variances } \\
\text { assumed }\end{array}$ & 22.26 & .000 & -9.25 & 490 & .000 & -1.78 & .19 & -2.16 & -1.40 \\
\hline & $\begin{array}{c}\text { Equal variances } \\
\text { not assumed }\end{array}$ & & & -9.26 & 462.78 & .000 & -1.78 & .19 & -2.16 & -1.40 \\
\hline \multirow[t]{2}{*}{$x 13$} & $\begin{array}{c}\text { Equal variances } \\
\text { assumed }\end{array}$ & 13.60 & .000 & -13.44 & 488 & .000 & -2.75 & .20 & -3.16 & -2.35 \\
\hline & $\begin{array}{c}\text { Equal variances } \\
\text { not assumed }\end{array}$ & & & -13.46 & 477.30 & .000 & -2.75 & .20 & -3.16 & -2.35 \\
\hline \multirow[t]{2}{*}{ x14 } & $\begin{array}{l}\text { Equal variances } \\
\text { assumed }\end{array}$ & 33.29 & .000 & -14.04 & 487 & .000 & -2.89 & .20 & -3.29 & -2.48 \\
\hline & $\begin{array}{l}\text { Equal variances } \\
\text { not assumed }\end{array}$ & & & -14.07 & 463.88 & .000 & -2.89 & .20 & -3.29 & -2.48 \\
\hline x15 & $\begin{array}{l}\text { Equal variances } \\
\text { assumed }\end{array}$ & 11.92 & .001 & -12.36 & 489 & .000 & -2.54 & .20 & -2.95 & -2.14 \\
\hline
\end{tabular}




\begin{tabular}{|c|c|c|c|c|c|c|c|c|c|c|}
\hline & \multirow[b]{4}{*}{$\begin{array}{l}\text { Equal variances } \\
\text { not assumed }\end{array}$} & \multicolumn{2}{|c|}{$\begin{array}{c}\text { Levene's Test for } \\
\text { Equality of } \\
\text { Variances }\end{array}$} & \multicolumn{7}{|c|}{ t-test for Equality of Means } \\
\hline & & \multirow[t]{2}{*}{$\mathrm{F}$} & \multirow[t]{2}{*}{ Sig. } & \multirow[t]{2}{*}{$\mathrm{t}$} & \multirow[t]{2}{*}{ df } & \multirow[t]{2}{*}{$\begin{array}{c}\text { Sig. } \\
\text { (2-tailed) }\end{array}$} & \multirow[t]{2}{*}{$\begin{array}{c}\text { Mean } \\
\text { Difference }\end{array}$} & \multirow[t]{2}{*}{$\begin{array}{l}\text { Std. Error } \\
\text { Difference }\end{array}$} & \multicolumn{2}{|c|}{$\begin{array}{c}95 \% \text { Confidence } \\
\text { Interval of the } \\
\text { Difference }\end{array}$} \\
\hline & & & & & & & & & Lower & Upper \\
\hline & & & & -12.37 & 480.18 & .000 & -2.54 & .20 & -2.95 & -2.14 \\
\hline \multirow[t]{2}{*}{$x 16$} & $\begin{array}{l}\text { Equal variances } \\
\text { assumed }\end{array}$ & 8.23 & .004 & -8.37 & 487 & .000 & -1.52 & .18 & -1.87 & -1.16 \\
\hline & $\begin{array}{l}\text { Equal variances } \\
\text { not assumed }\end{array}$ & & & -8.39 & 468.45 & .000 & -1.52 & .18 & -1.87 & -1.16 \\
\hline \multirow[t]{2}{*}{$\mathrm{x} 17$} & $\begin{array}{l}\text { Equal variances } \\
\text { assumed }\end{array}$ & .31 & .577 & -4.56 & 490 & .000 & -.85 & .18 & -1.21 & -.48 \\
\hline & $\begin{array}{l}\text { Equal variances } \\
\text { not assumed }\end{array}$ & & & -4.56 & 488.10 & .000 & -.85 & .18 & -1.21 & -.48 \\
\hline \multirow[t]{2}{*}{$x 18$} & $\begin{array}{l}\text { Equal variances } \\
\text { assumed }\end{array}$ & 3.22 & .073 & -7.17 & 492 & .000 & -1.27 & .17 & -1.62 & -.92 \\
\hline & $\begin{array}{l}\text { Equal variances } \\
\text { not assumed }\end{array}$ & & & -7.17 & 485.15 & .000 & -1.27 & .17 & -1.62 & -.92 \\
\hline \multirow[t]{2}{*}{$x 19$} & $\begin{array}{l}\text { Equal variances } \\
\text { assumed }\end{array}$ & 61.19 & .000 & -15.25 & 489 & .000 & -3.49 & .22 & -3.94 & -3.04 \\
\hline & $\begin{array}{l}\text { Equal variances } \\
\text { not assumed }\end{array}$ & & & -15.27 & 456.70 & .000 & -3.49 & .22 & -3.94 & -3.04 \\
\hline \multirow[t]{2}{*}{$x 20$} & $\begin{array}{l}\text { Equal variances } \\
\text { assumed }\end{array}$ & 65.69 & .000 & -18.34 & 488 & .000 & -4.07 & .22 & -4.51 & -3.63 \\
\hline & $\begin{array}{l}\text { Equal variances } \\
\text { not assumed }\end{array}$ & & & -18.37 & 460.50 & .000 & -4.07 & .22 & -4.50 & -3.63 \\
\hline \multirow[t]{2}{*}{$x 21$} & $\begin{array}{l}\text { Equal variances } \\
\text { assumed }\end{array}$ & 75.02 & .000 & -8.56 & 486 & .000 & -2.25 & .26 & -2.76 & -1.73 \\
\hline & $\begin{array}{l}\text { Equal variances } \\
\text { not assumed }\end{array}$ & & & -8.58 & 426.61 & .000 & -2.25 & .26 & -2.76 & -1.73 \\
\hline \multirow[t]{2}{*}{$x 22$} & $\begin{array}{l}\text { Equal variances } \\
\text { assumed }\end{array}$ & 3.50 & .062 & -3.48 & 483 & .001 & -.77 & .22 & -1.20 & -.33 \\
\hline & $\begin{array}{l}\text { Equal variances } \\
\text { not assumed }\end{array}$ & & & -3.47 & 470.89 & .001 & -.77 & .22 & -1.20 & -.33 \\
\hline \multirow[t]{2}{*}{$x 23$} & $\begin{array}{l}\text { Equal variances } \\
\text { assumed }\end{array}$ & .74 & .389 & -7.48 & 491 & .000 & -1.48 & .19 & -1.87 & -1.09 \\
\hline & $\begin{array}{l}\text { Equal variances } \\
\text { not assumed }\end{array}$ & & & -7.48 & 490.84 & .000 & -1.48 & .19 & -1.87 & -1.09 \\
\hline \multirow[t]{2}{*}{$x 24$} & $\begin{array}{l}\text { Equal variances } \\
\text { assumed }\end{array}$ & 3.61 & .058 & -7.88 & 492 & .000 & -1.55 & .19 & -1.94 & -1.16 \\
\hline & $\begin{array}{l}\text { Equal variances } \\
\text { not assumed }\end{array}$ & & & -7.88 & 480.51 & .000 & -1.55 & .19 & -1.94 & -1.16 \\
\hline \multirow[t]{2}{*}{$\mathrm{x} 25$} & $\begin{array}{l}\text { Equal variances } \\
\text { assumed }\end{array}$ & 1.16 & .281 & -4.06 & 486 & .000 & -.75 & .18 & -1.11 & -.38 \\
\hline & $\begin{array}{l}\text { Equal variances } \\
\text { not assumed }\end{array}$ & & & -4.07 & 481.18 & .000 & -.75 & .18 & -1.11 & -.38 \\
\hline \multirow[t]{2}{*}{$x 26$} & $\begin{array}{l}\text { Equal variances } \\
\text { assumed }\end{array}$ & 23.45 & .000 & -14.67 & 489 & .000 & -3.29 & .22 & -3.73 & -2.85 \\
\hline & $\begin{array}{l}\text { Equal variances } \\
\text { not assumed }\end{array}$ & & & -14.68 & 474.82 & .000 & -3.29 & .22 & -3.73 & -2.85 \\
\hline \multirow[t]{2}{*}{$x 27$} & $\begin{array}{l}\text { Equal variances } \\
\text { assumed }\end{array}$ & .85 & .355 & -5.98 & 489 & .000 & -1.32 & .22 & -1.75 & -.88 \\
\hline & $\begin{array}{l}\text { Equal variances } \\
\text { not assumed }\end{array}$ & & & -5.98 & 488.11 & .000 & -1.32 & .22 & -1.75 & -.88 \\
\hline $\mathrm{x} 28$ & $\begin{array}{l}\text { Equal variances } \\
\text { assumed }\end{array}$ & 2.31 & .129 & -6.17 & 488 & .000 & -1.40 & .22 & -1.85 & -.95 \\
\hline
\end{tabular}


Comparative Analysis of Innovation Capability Indexes

\begin{tabular}{|c|c|c|c|c|c|c|c|c|c|c|}
\hline & & \multicolumn{2}{|c|}{$\begin{array}{l}\text { Levene's Test for } \\
\text { Equality of } \\
\text { Variances }\end{array}$} & \multicolumn{7}{|c|}{ t-test for Equality of Means } \\
\hline & & \multirow[t]{2}{*}{$\mathrm{F}$} & \multirow[t]{2}{*}{ Sig. } & \multirow[t]{2}{*}{$\mathrm{t}$} & \multirow[t]{2}{*}{$\mathrm{df}$} & \multirow[t]{2}{*}{$\begin{array}{c}\text { Sig. } \\
\text { (2-tailed) }\end{array}$} & \multirow[t]{2}{*}{$\begin{array}{c}\text { Mean } \\
\text { Difference }\end{array}$} & \multirow[t]{2}{*}{$\begin{array}{l}\text { Std. Error } \\
\text { Difference }\end{array}$} & \multicolumn{2}{|c|}{$\begin{array}{l}95 \% \text { Confidence } \\
\text { Interval of the } \\
\text { Difference }\end{array}$} \\
\hline & & & & & & & & & Lower & Upper \\
\hline & $\begin{array}{l}\text { Equal variances } \\
\text { not assumed }\end{array}$ & & & -6.17 & 487.96 & .000 & -1.40 & .22 & -1.85 & -.95 \\
\hline \multirow[t]{2}{*}{ x29 } & $\begin{array}{l}\text { Equal variances } \\
\text { assumed }\end{array}$ & 31.99 & .000 & -18.29 & 487 & .000 & -3.94 & .21 & -4.37 & -3.52 \\
\hline & $\begin{array}{l}\text { Equal variances } \\
\text { not assumed }\end{array}$ & & & -18.33 & 472.41 & .000 & -3.94 & .21 & -4.37 & -3.52 \\
\hline \multirow[t]{2}{*}{$\mathrm{x} 30$} & $\begin{array}{l}\text { Equal variances } \\
\text { assumed }\end{array}$ & 9.14 & .003 & -17.22 & 483 & .000 & -3.79 & .22 & -4.22 & -3.35 \\
\hline & $\begin{array}{l}\text { Equal variances } \\
\text { not assumed }\end{array}$ & & & -17.26 & 479.46 & .000 & -3.79 & .21 & -4.22 & -3.36 \\
\hline \multirow[t]{2}{*}{$\mathrm{x} 31$} & $\begin{array}{l}\text { Equal variances } \\
\text { assumed }\end{array}$ & 15.59 & .000 & -19.14 & 486 & .000 & -4.22 & .22 & -4.65 & -3.79 \\
\hline & $\begin{array}{l}\text { Equal variances } \\
\text { not assumed }\end{array}$ & & & -19.17 & 478.25 & .000 & -4.22 & .22 & -4.65 & -3.79 \\
\hline \multirow[t]{2}{*}{$\mathrm{x} 32$} & $\begin{array}{l}\text { Equal variances } \\
\text { assumed }\end{array}$ & 20.77 & .000 & -18.74 & 488 & .000 & -4.14 & .22 & -4.58 & -3.71 \\
\hline & $\begin{array}{l}\text { Equal variances } \\
\text { not assumed }\end{array}$ & & & -18.76 & 477.53 & .000 & -4.14 & .22 & -4.58 & -3.71 \\
\hline \multirow[t]{2}{*}{$\mathrm{x} 33$} & $\begin{array}{l}\text { Equal variances } \\
\text { assumed }\end{array}$ & 9.62 & .002 & -1.78 & 489 & .075 & -.37 & .20 & -.78 & .03 \\
\hline & $\begin{array}{l}\text { Equal variances } \\
\text { not assumed }\end{array}$ & & & -1.78 & 478.06 & .075 & -.37 & .20 & -.78 & .03 \\
\hline \multirow[t]{2}{*}{ x34 } & $\begin{array}{l}\text { Equal variances } \\
\text { assumed }\end{array}$ & .66 & .416 & -12.13 & 485 & .000 & -2.44 & .20 & -2.84 & -2.04 \\
\hline & $\begin{array}{l}\text { Equal variances } \\
\text { not assumed }\end{array}$ & & & -12.14 & 484.66 & .000 & -2.44 & .20 & -2.84 & -2.05 \\
\hline \multirow[t]{2}{*}{ x35 } & $\begin{array}{l}\text { Equal variances } \\
\text { assumed }\end{array}$ & 9.09 & .003 & -4.93 & 487 & .000 & -1.04 & .21 & -1.45 & -.62 \\
\hline & $\begin{array}{l}\text { Equal variances } \\
\text { not assumed }\end{array}$ & & & -4.93 & 475.39 & .000 & -1.04 & .21 & -1.45 & -.62 \\
\hline \multirow[t]{2}{*}{$\mathrm{x} 36$} & $\begin{array}{l}\text { Equal variances } \\
\text { assumed }\end{array}$ & 9.30 & .002 & -5.04 & 488 & .000 & -1.08 & .21 & -1.50 & -.66 \\
\hline & $\begin{array}{l}\text { Equal variances } \\
\text { not assumed }\end{array}$ & & & -5.05 & 471.75 & .000 & -1.08 & .21 & -1.50 & -.66 \\
\hline \multirow[t]{2}{*}{ x37 } & $\begin{array}{l}\text { Equal variances } \\
\text { assumed }\end{array}$ & 19.87 & .000 & -6.07 & 491 & .000 & -1.28 & .21 & -1.69 & -.86 \\
\hline & $\begin{array}{l}\text { Equal variances } \\
\text { not assumed }\end{array}$ & & & -6.08 & 460.67 & .000 & -1.28 & .21 & -1.69 & -.86 \\
\hline \multirow[t]{2}{*}{ x38 } & $\begin{array}{l}\text { Equal variances } \\
\text { assumed }\end{array}$ & 9.88 & .002 & -2.10 & 488 & .036 & -.46 & .21 & -.89 & -.03 \\
\hline & $\begin{array}{l}\text { Equal variances } \\
\text { not assumed }\end{array}$ & & & -2.11 & 470.54 & .035 & -.46 & .21 & -.89 & -.03 \\
\hline \multirow[t]{2}{*}{ x39 } & $\begin{array}{l}\text { Equal variances } \\
\text { assumed }\end{array}$ & 6.18 & .013 & -.92 & 487 & .356 & -.20 & .22 & -.63 & .22 \\
\hline & $\begin{array}{l}\text { Equal variances } \\
\text { not assumed }\end{array}$ & & & -.92 & 471.11 & .355 & -.20 & .22 & -.63 & .22 \\
\hline \multirow[t]{2}{*}{$\mathrm{x} 40$} & $\begin{array}{l}\text { Equal variances } \\
\text { assumed }\end{array}$ & 27.04 & .000 & -12.33 & 489 & .000 & -2.95 & .23 & -3.42 & -2.48 \\
\hline & $\begin{array}{l}\text { Equal variances } \\
\text { not assumed }\end{array}$ & & & -12.34 & 473.08 & .000 & -2.95 & .23 & -3.41 & -2.48 \\
\hline
\end{tabular}


As Table 3 indicates, the significance level in all cases except eighth, ninth, eleventh, and seventeenth indexes is smaller than 0.5. Therefore, the assumption of equal variances is rejected and the information contained in the second row is analyzed to measure the mean of indexes (with the exception of the eighth, ninth, eleventh, and seventeenth indexes). In all cases estimated sig is .000 and less than 0.05 ; hence, there is a significant difference between the existing and desired status. Also, because of the negative result of lower and higher bound in all cases except the $39^{\text {th }}$, the assessment is reported unfavorable and for the $39^{\text {th }}$ index the situation is reported to be ordinary.

\section{Results}

This study aimed to examine the gaps of innovation capability factors in ESCO. To this end, Charles Cochran formula was used to calculate 247 as sample size with 0.5 per cent margin of error. The questionnaire was developed and distributed among company experts. Then, they were analyzed as shown in Table 4. To calculate the desired level, the idea of steel industry experts was used. Cronbach's alpha was 0.94 which shows the reliability of the questionnaire.

Table 4

The Frequency of the Experts

\begin{tabular}{cccc}
\hline Position & Frequency & Frequency (\%) & Cumulative Frequency \\
\hline Change Management Expert & 42 & 17 & 17 \\
Supporting Expert & 24 & 9.7 & 26.7 \\
Human Resources Expert & 23 & 9.3 & 36 \\
BSc in Health Environmental & 22 & 8.9 & 44.9 \\
BS in Budget & 23 & 9.3 & 54.3 \\
Manufacture Expert & 27 & 10.9 & 65.2 \\
Sales Expert & 28 & 11.3 & 76.5 \\
Expert of Quality Control & 30 & 12.1 & 88.7 \\
Steel Expert & 8 & 3.2 & 91.9 \\
BS in Statistics and Accounting & 8 & 3.2 & 95.1 \\
Managements & 12 & 4.9 & 100 \\
Sum & 247 & 100 & -- \\
\hline
\end{tabular}

\section{Discussion and Conclusion}

The aim of the study was to investigate the identification and comparison of structural factors of innovation capability in ESCO with desired situation. Evaluation of the innovation capability factors of ESCO compared with its desired status in industry can help companies develop innovative strategies and also can assist them to achieve organizational goals. Based 
on the data analysis and findings of this study, it can be concluded that existing statuses of innovation capability was unfavorable against desired statuses in ESCO and all cases except the $39^{\text {th }}$ were reported unfavorable.

Based on the results of this study, applications in accordance with statuses will be provided as follows. Firstly, about the most important index in the group dimension, teamwork and problem-solving mechanisms, teaching working groups for innovation and learning problem solving mechanisms is recommended. Secondly, regarding the index of promotion and reward systems and the support of innovators, it is suggested to consider supported sources in innovators and providers of innovative ideas as promotions and bonuses. In this regard, the suggestions systems can be effective. In addition to this, for the weakness of structure, leadership and performance of managers, speed management in process of innovative ideas and delegating to the respective units is recommended. Also, indexes related to problems arising from financing, such as the resources allocated to the process of innovation, research and development budget, and dependence on other sources can be suggested in the formulation of the company's research funds. In this respect, budget of this unit can be specified. Besides, according to the results, for the weakness in the index of creativity in methods of operation, number of patents and time allocated for innovative ideas, lack of awareness of staff and the system plays an important role in this matter.

Therefore, in conclusion, training and creating awareness in the company is recommended. Also, it is imperative to stress that establishing technology and innovation management unit should be taken into consideration.

\section{References}

Akman, G., \& Yilmaz, C. (2008). Innovation capacity, innovation strategy and market orientation: An empirical analysis in Turkish software industry. International Journal of Innovation Management, 12(1), 69-111.

Arasti, M. R., Karamipour, A., \& Quraishi, B. (2009). Identifying the factors affecting the innovation capacity of enterprises: A case study of Iranian industrial automation companies. Journal of Management Science, 4(15), 1-30.

Bertland, O. (2009). Effects of foreign acquisitions on R \& D activity: Evidence from firm-level data for France. Research Policy, 38(6), 1021-1031.

Forsman, H. (2011). Innovation capacity and innovation development in small enterprises: A comparison between the manufacturing and service sectors. Research Policy, 40(5), 739-750.

Hull, C. E., \& Covin, J. G. (2010). Learning capability, technological parity, and innovation mode use. Journal of Product Innovation Management, 27(1), 97-114.

Khalil, T. (2000). Management of Technology: The key to competitiveness and wealth creation. USA: McGraw-Hill.

Kroll, H., \& Schiller, D. (2010). Establishing an interface between public sector applied research and the Chinese enterprise sector: Preparing for 2020. Technovation, 30(2), 117-129.

Nematolahi, F. (2011). Designing a framework of Assessment innovation in the product: A case study of mechanical products (Unpublished MSA thesis), Tehran University, Tehran. 
Pakzad, M., \& Tabatabaeian, H. (2005). Assessment of Innovation survey systems and provide a framework for measuring innovation in Iran. Modarres Human Sciences, 10(1), 161-190.

Roman, M. A., Gamero, J., \& Tamayo, J. A. (2011). Analysis of innovation in SMEs using an innovative capability-based non- linear model: A study in the province of Seville (Spain). Techovation, 31(9), 1-17.

Saunila, M., Ukko, J., \& Rantanen, H. (2014). Does innovation capability really matter for the profitability of SMEs? Journal of Corporate Transformation, 21(2), 134-142.

Shekarchizadeh, A. (2011). Identify indicators for evaluating the innovation management in education industry (Unpublished MSA thesis), Tehran University, Tehran.

Tidd, J., \& Bessant, T. (2009). Managing Innovation: Integrating technological, market and organizational change. $\left(3^{\text {rd }}\right.$ ed.).USA: John Wiley and Sons.

Yang, J. (2012). Innovation capability \& corporate growth: An empirical investigation in China. Journal of Engineering \& Technology Management, 29(1), 34-46.

Yeşil, S. (2013). Exploring the link between knowledge sharing enablers, innovation capability and innovation performance. International Journal of Innovation Management, 17(4), 246-266. 